\title{
The Effect of Supplementing a Novel n-3 Polyunsaturated Fatty Acids Formulation Containing Schisandra chinensis Extract and Vitamin D3 to a Group of Elite Sport Athletes on Competition Related Stress, Anxiety and Self-confidence
}

\author{
${ }^{1}$ Andrea Belluzzi", ${ }^{2}$ Peter Lembke, ${ }^{1}$ Giovanni Marasco, ${ }^{1}$ Eleonora Scaioli \\ ${ }^{1}$ Department of Medical and Surgical Sciences, Sant'Orsola-Malpighi Hospital, Bologna, Italy. ${ }^{2}$ Paseo de \\ las Delicias 30, Madrid, Spain.
}

Submitted 05 September 2019; Accepted in final form 02 November 2019.

\begin{abstract}
Background. Competitive sport has the potential for high levels of stress and anxiety that may affect performance and increase the risk of injuries. While benefits of pre-training assumption of N-3 polyunsaturated fatty acids are well known, poor data are available on Schisandra chinensis. Schisandra chinensis is a medicinal plant in use in Chinese medicine which increases physical working capacity and affords a stress protective effect. Objectives. We tested, in an open pilot study, the effect on competition related stress, anxiety and self-confidence of a novel formulation containing: PUFAs, Schisandra chinensis extract and vitamin D3. Methods. A novel formulation containing N-3 polyunsaturated fatty acids (PUFAs), Schisandra chinensis extract and vitamin D3, five ml per day was administered to one group of fifteen elite sport athletes (basketball players). Competitive State Anxiety Inventory-2 (CSAI-2) tests were administered at baseline and after 3 months. Results. All subjects completed the 3 months period of the study, all declaring a consumption $>75 \%$ of daily recommended dose. No adverse event were registered. After intervention, the median cognitive state anxiety score remained mild (score 16) even if with an overall statistically significative decrease $(\mathrm{p}=0.005)$; same results were registered after intervention for the somatic state anxiety score (score 16) with an overall statistically significative decrease $(\mathrm{p}=0.008)$ and for the self-confidence score (score 22 , range 15-30) with an overall statistically significative increase $(\mathrm{p}=0.008)$. Conclusion. The results showed the positive effect of the treatment on the control of stress and anxiety and the improvement of self-confidence in a large majority of the subject enrolled. However, further studies are needed to confirm our findings.
\end{abstract}

\section{KEYWORDS: Omega 3, Sport Supplementation, Sport Psychology}

\section{INTRODUCTION}

Not surprisingly, competitive sport has the potential for high levels of stress and anxiety. Despite that some amount of anxiety may facilitate performance, generally excessive sportrelated anxiety is associated with outcome in elite athletes including the risk of injuries (1). Different approach such as psychological therapy are in use to combat such a status that can profoundly impact on performance. High mental toughness, defined as the ability to achieve high levels of subjective or objective performance facing obstacles and adversity (2), may produce high performance and health benefit to athletes. N-3 polyunsaturated fatty acids (PUFAs) supplementation deeply influences psychological process (3). In rats level of dopamine increases,

*. Corresponding Author:

Andrea Belluzzi, MD

E-mail: andrea.belluzzi@aosp.bo.it 
during Eicosapentaenoic acid (EPA) supplementation, protecting against mood disorders such as anxiety (4). Eicosapentaenoic and docosahexaenoic acid (DHA) supplementation has shown to improve the Profile of Mood States (POMS) in sport students after heavy eccentric exercise (5). N-3 PUFAS has been shown to induce bradycardia by reducing the catecholamine levels (6) deeply involved in the development of anxiety (7). Moreover very recent studies underlined the role of n-3 PUFAs in controlling the inflammatory processes and in favoring the resolution of trauma injuries in athletes by releasing specialized pro-resolvins mediators (SPMs) (8). It has been shown that supplementation with n-3 PUFAs during preseason training results in reduced muscle soreness and injuries and favor a quicker recovery from trauma (9).

Schisandra chinensis is a medicinal plant (berry) in use in Chinese medicine and well established in Russia where it has been recently included in their State Drug Register. The active biological principle is schizandrin, belonging to the lignans, a large group of polyphenols found in plants. Schisandra increases physical working capacity and affords a stress protective effect against a broad spectrum of harmful factors including heat shock, skin burns, cooling etc. The effects on central nervous system include increases of endurance, accuracy of movement, mental performance being effective on asthenia, psychogenic depression, astheno-depressive states, hypotension etc (10).

Schisandrins are lipophilic, so they easily dissolve in Omega-3 oils. Such a lipophilic Schisandra extract is more bioavailable than the standard water/alcohol based formulations and thus a combination of these two natural active ingredients may be of advantage. The same logic was applied for the Vitamin D3 - which, among others, is also known to support and maintain a positive mindset, especially if a vitamin $D$ deficiency is present (11).

In this pilot open study we intended to test the effect of n-3 PUFAS, Schisandra and Vitamin D3 enriched preparation in a liquid form as supplement on cognitive state anxiety, somatic state anxiety and self-confidence defined accordingly to Competitive State Anxiety Inventory-2 (CSAI-2) test (12) that is one of the most frequently used and validated instruments when assessing competitive state anxiety in sport psychology research (13).

\section{MATERIALS AND METHODS}

Participants. A group of fifteen men elite professional basketball players ranging from 18 to 36 years agreed to participate. A written informed consent was obtained from all subjects enrolled in the study in accordance with the principles of the Declaration of Helsinki (revision of Edinburgh 2000).

Research Design. From December 2017 to June 2018, all the athletes enrolled were supplemented for a three months period with a dose of $5 \mathrm{ml}$ per day of Omega Complex ${ }^{\mathrm{TM}}$, accounting for $1.125 \mathrm{~g}$ of $\mathrm{n}-3$ PUFAs (EPA+DHA), $200 \mathrm{mg}$ of Schisandra chinensis extract (containing approximately $16 \mathrm{mg}$ schisandrins (Lignans)) - and $15 \mu \mathrm{g}$ Vitamin D3 (Table 1). The dose was the same for each athlete, the median weight value was $99.5 \mathrm{Kg}$ (88-113). For all the athletes included the BMI was normal, ranging from 18.5 to 24.9 .

The mixture was in a liquid form and compliance was measure by empty bottles returned every month by the athletes. A declared consumption of $>75 \%$ of the dose suggested was considered adequate. Competitive State Anxiety Inventory-2 (CSAI-2) tests (12) were administered at baseline and after 3 months of supplementation.

Table 1. Omega-3 Complex ${ }^{\mathrm{TM}}$ composition (5 ml)

\begin{tabular}{|lc|}
\hline Total Omega-3 & $1315 \mathrm{mg}$ \\
Eicosapentaenoic acid (EPA) & $690 \mathrm{mg}$ \\
Docosahexaenoic acid (DHA) & $435 \mathrm{mg}$ \\
Schisandra cinensis extract & $200 \mathrm{mg}$ \\
Vitamin D3 & $15 \mu \mathrm{g}$ \\
\hline
\end{tabular}

Measures. Competitive State Anxiety Inventory-2 (CSAI-2) tests (12) (Table 2) were administered at baseline and after 3 month of supplementation with Omega Complex ${ }^{\mathrm{TM}}$, accordingly to this instructions "Complete the following scale on two separate occasions: during a quiet time before practice when you are fairly relaxed, and during a competitive situation that you feel is highly stressful."

To score the CSAI-2, we took all the scores for each item at face values with the exception of item 14 , were we "reverse" the score.

All validated questionaries' answers were analyzed by the authors and translated into numerical scores. Then, the results were 
grouped into the 3 categories above cited (cognitive state anxiety score, somatic state anxiety score and self-confidence score). For each category scores patients with a score from 9 to 14 were classified as having lowrisk, from 15 to 23 as having mild-risk and for score above 23 as having high-risk for cognitive or somatic anxiety. For the selfconfidence level, the results are inversely interpretation ranging from low selfconfidence to high self-confidence. After the supplement intervention, the patients repeated the questionnaire.

Statistical Analysis. CSAI's score were expressed as continuous variables as median (range). For group comparison, a two-sample Wilcoxon rank-sum (Mann-Whitney) test was used to compare baseline versus post intervention scores results. All analyses were carried out using STATA 13 statistical software (Stata Corp., College Station, TX, USA).

Table 2. Competitive State Anxiety Inventory-2 (CSAI-2) test

\begin{tabular}{|c|c|c|c|c|}
\hline & Not at all & Somewhat & Moderately & Very much \\
\hline 1. I am concerned about this competition. & 1 & 2 & 3 & 4 \\
\hline 2. I feel nervous. & 1 & 2 & 3 & 4 \\
\hline 3. I feel at ease. & 1 & 2 & 3 & 4 \\
\hline 4. I have self-doubts. & 1 & 2 & 3 & 4 \\
\hline 5. I feel jittery. & 1 & 2 & 3 & 4 \\
\hline 6. I feel comfortable. & 1 & 2 & 3 & 4 \\
\hline 7. I am concerned I may not do as well in this competition as I could. & 1 & 2 & 3 & 4 \\
\hline 8. My body feels tense. & 1 & 2 & 3 & 4 \\
\hline 9. I feel self-confident. & 1 & 2 & 3 & 4 \\
\hline 10. I am concerned about losing. & 1 & 2 & 3 & 4 \\
\hline 11. I feel tense in my stomach. & 1 & 2 & 3 & 4 \\
\hline 12. I feel secure. & 1 & 2 & 3 & 4 \\
\hline 13. I am concerned about losing. & 1 & 2 & 3 & 4 \\
\hline 14. My body feels relaxed. & 1 & 2 & 3 & 4 \\
\hline 15. I'm confident I can meet the challenge. & 1 & 2 & 3 & 4 \\
\hline 16. I'm concerned about performing poorly. & 1 & 2 & 3 & 4 \\
\hline 17. My heart is racing. & 1 & 2 & 3 & 4 \\
\hline 18. I'm confident about performing well. & 1 & 2 & 3 & 4 \\
\hline 19. I'm worried about reaching my goal. & 1 & 2 & 3 & 4 \\
\hline 20. I feel my stomach sinking. & 1 & 2 & 3 & 4 \\
\hline 21. I feel mentally relaxed. & 1 & 2 & 3 & 4 \\
\hline 22. I'm concerned that others will be disappointed with my performance. & 1 & 2 & 3 & 4 \\
\hline 23. My hands are clammy. & 1 & 2 & 3 & 4 \\
\hline 24. I'm confident because I mentally picture myself reaching my goal. & 1 & 2 & 3 & 4 \\
\hline 25. I'm concerned I won't be able to concentrate. & 1 & 2 & 3 & 4 \\
\hline 26. My body feels tight. & 1 & 2 & 3 & 4 \\
\hline 27. I'm confident of coming through under pressure. & 1 & 2 & 3 & 4 \\
\hline
\end{tabular}

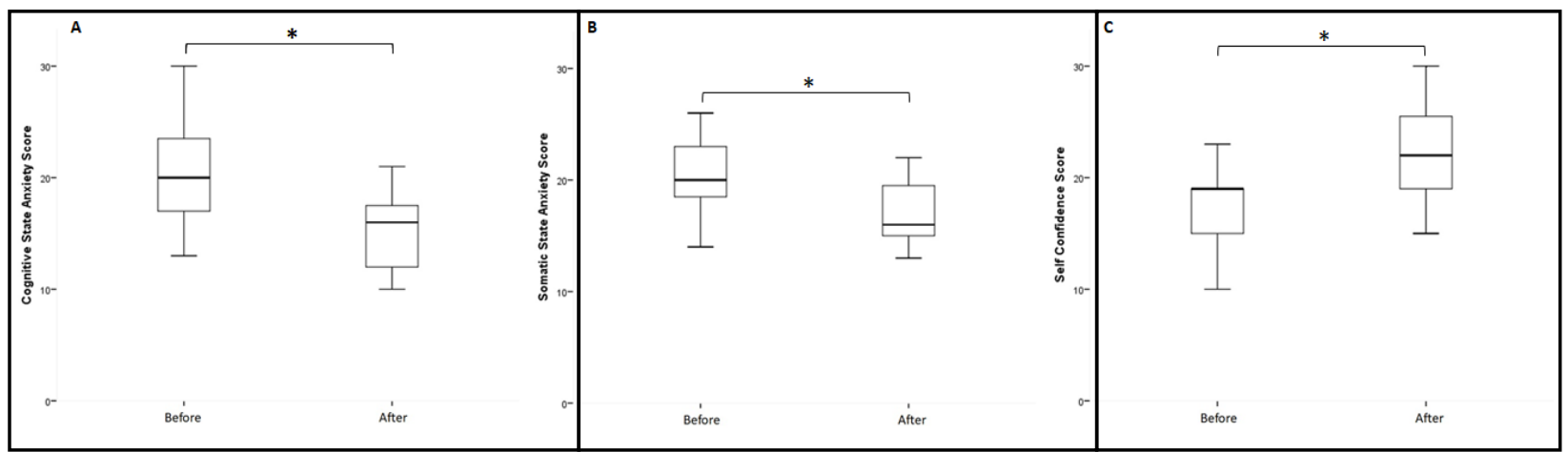

Figure 1. Box Plots for Illinois Competition Test Score before and after Intervention According to each Domain: A) Cognitive State Anxiety; B) Somatic State Anxiety; C) Self-confidence; *: p<0.05 at two-sample Wilcoxon Rank-sum (Mann-Whitney) Test.

\section{RESULTS}

All subjects completed the 3 months period of the study, all declaring a consumption $>75 \%$ of daily recommended dose. No adverse event were registered. All subjects completed the CSAI-2 questionnaire. The CSAI-2 demonstrated high internal consistency $($ Cronback's alfa $=0.91)$.

The median baseline cognitive state anxiety score reported a mild level (score 20, range 1331) of cognitive state anxiety; in particular 4 
subjects reported a high CSA level, 10 subjects a mild CSA level and one subject a low CSA level. After intervention, the median CSA score reported remained mild (score 16, range 10-21) even if with an overall statistically significative decrease $(\mathrm{p}=0.005)$ (Figure $1 \mathrm{~A})$; in particular, after intervention no subjects reported a high CSA level, 9 subjects reported a mild CSA level and 6 subjects reported a low CSA level. All subjects who report an improvement experienced a highmild or mild-low CSA score improvement, except for one subject which reported a high-low CSA score improvement.

The median baseline somatic state anxiety (SSA) score reported a mild level (score 20, range 14-26) of somatic state anxiety; in particular 4 subjects reported a high SSA level, 10 subjects a mild SSA level and one subject a low SSA level. After intervention, the median SSA score reported remained mild (score 16, range 13-22) even if with an overall statistically significative decrease $(p=0.008)$ (Figure $1 \mathrm{~B})$; in particular, after intervention no subjects reported a high SSA level, 12 subjects reported a mild SSA level and 3 subjects reported a low SSA level. All subjects who report an improvement experienced a highmild or mild-low SSA score improvement.

The median baseline self-confidence (SC) score reported a mild level (score 19, range 1023) of self-confidence; in particular no subjects reported a high SC level, 14 subjects a mild SC level and one subject a low SC level. After intervention, the median SC score reported remained mild (score 22, range 15-30) even if with an overall statistically significative increase $(\mathrm{p}=0.008) \quad$ (Figure 1C); in particular, after intervention 6 subjects reported a high SC level, 9 subjects reported a mild SC level and no subjects reported a low SC level. All subjects who report an improvement experienced a mild-high or low-mild SC score improvement, except for one subject which reported a low-high SC score improvement.

\section{DISCUSSION}

This open pilot study shows the positive effect of a mixture of n-3 PUFAs, Schisandra chinensis plant extract and Vitamin D3 on the control of stress and anxiety in a group of elite competing basketball players.

Western diet is an extremely poor source of n3 PUFAs, even in the Mediterranean countries like for example Italy, Spain and France. Studies reported that the consumption of n-3 PUFAs in the diet, declined in the last decades and that the daily amount of n-3 PUFAs consumption did not reach the WHO suggested levels for a healthy diet: this being especially the case for the young population, the main resource for competing sport (14). Available evidence confirms that athletes show low levels of n-3 PUFAs in their blood (15-18).

More recently, Wilson et al. (19) investigated if dietary intake of n-3 PUFAs would be negatively associated with competitive and general anxiety and positively associated with mental toughness. Whole blood and dietary levels of EPA, docosapentaenoic acid (DPA), DHA were modestly negatively associated with general but not sport-specific anxiety in female in American collegiate athletes during a pre-season period. DPA blood level showed a significative association with mental toughness. These data, although preliminary, suggest that n-3 PUFAs status may influence general anxiety and resilience in athletes.

Lembke et al. (5) showed that a daily supplementation of $2.7 \mathrm{~g}$ EPA + DHA in the ethyl-ester form (EE), over a period of 30 days, improved significantly the Profile of Mood States (POMS) score in sport students after heavy eccentric exercise. These students felt less muscle soreness and had a more positive mindset compared to the placebo group, thus suggesting a positive influence on their athletic performance.

In our study the athletes supplemented their normal daily diet, during the 3 months study, with $5 \mathrm{ml}$ Omega-3 Complex, containing $1.125 \mathrm{~g}$ EPA + DHA (both in the highly bioavailable triglyceride form), $200 \mathrm{mg}$ Schisandra chinensis extract and $15 \mu \mathrm{g}$ vitamin D3.

Our results support a positive correlation between supplementation of an n-3 PUFAs / Schisandra chinensis extract / Vitamin D3 formulation and anxiety control also improving the "self-confidence" mental toughness.

In previous studies supplemented Schisandra chinensis extract has shown to be able to improve cognitive function resistance to fatigue and stress control, accuracy of movement, mental performance, and endurance performance. In fact, the main active principles of the plant, the Lignans or schisandrins, have been tested in those sport activities where anxiety control and concentration capacity is pivotal for a better performance, such as equestrian, shooting sport. 
In a placebo controlled double blind study, the effects of Schisandra extract on stress were tested in 185 athletes at the dose of $91 \mathrm{mg}$ extract/tablet, 2 tablets twice a daily, for 7 days quoting nitric oxide (NO) and cortisol level on saliva as an indirect adaptogen response to stress. The group of athletes included jumper, cyclist, boxers and weightlifters. The results showed that Schisandra tablets exhibited a pro-stressor effect activating the formation of $\mathrm{NO}$ and cortisol in both plasma and saliva and that such activation adapts to further heavy physical loading (20).

In our study, the dose of Schisandra chinensis extract present in the n-3 PUFAs oil was $200 \mathrm{mg}$ per day, corresponding to $16 \mathrm{mg}$ schisandrin + gamma-schisandrins. Compared to Panossian et al. (20), thus a slightly higher daily schisandrin dose combined with a much longer (3 months) intake.

The major limitation of the study is the open label design not accounting for an additional group of athletes with placebo assumption; however, this limit has been partially overcome with the comparison of basal CSAI-2 test made before intervention. Moreover, since CSAI-2 test was administered only twice, we did not test the learning effect for the test. Finally, another limitation of the study is the lack of information on n-3 PUFAs blood level before and after the supplementation; this would have add a great value of the results, possibly correlating low basal level on n-3 PUFAs with higher level of anxiety before the supplementation.

\section{CONCLUSION}

In conclusion, the preliminary results of this open pilot study showed a significant improvement on cognitive state anxiety, somatic state anxiety and self-confidence in a group of elite competing basketball players supplemented with a combination of n-3 PUFAs with Schisandra chinensis plant extract and Vitamin D3. This formulation may be of interest for improving athletic performance. The importance of the data provided here merits further investigation in well purposely designed, large, confirmatory studies.

\section{APPLICABLE REMARKS}

- Competing basketball players as other competing athletes show high level of cognitive state anxiety, somatic state anxiety and selfconfidence.

- Supplementation with a mixture of n-3 PUFAs and Schisandra chinensis extract plus vitamin D3, seem to have a positive impact and improve athletic performance.

\section{FINANCIAL DISCLOSURE}

None.

\section{FUNDING/SUPPORT}

None.

\section{AUTHORSHIP RIGHT}

$\mathrm{AB}$ and PL performed the study concept and design and the acquisition of the study data. GM performed the statistical analysis. AB, ES, GM and PL wrote the initial draft of the manuscript and did the final approval.

\section{ACKNOWLEDGEMENTS}

The authors would like to thank Beps Biopharm S.L/Spain for the supply of The Omega-3 Complex (Puro Omega ${ }^{\mathrm{R}}$ ).

\section{CONFLICT OF INTEREST}

The authors declare that they have no competing interests.

\section{REFERENCES}

1. Johnson U. Psychosocial antecedents of sport injury, prevention, and intervention: An overview of theoretical approaches and empirical findings. Int J Sport Exercise Psychol. 2007;5(4):352-369. doi: 10.1080/1612197x.2007.9671841

2. Gucciardi DF, Hanton S, Gordon S, Mallett CJ, Temby P. The concept of mental toughness: tests of dimensionality, nomological network, and traitness. J Pers. 2015;83(1):26-44. doi: 10.1111/jopy.12079 pmid: 24428736

3. Agostoni C, Nobile M, Ciappolino V, Delvecchio G, Tesei A, Turolo S, et al. The Role of Omega3 Fatty Acids in Developmental Psychopathology: A Systematic Review on Early Psychosis, Autism, and ADHD. Int J Mol Sci. 2017;18(12). doi: 10.3390/ijms18122608 pmid: 29207548

4. Song C, Li X, Kang Z, Kadotomi Y. Omega-3 fatty acid ethyl-eicosapentaenoate attenuates IL1beta-induced changes in dopamine and metabolites in the shell of the nucleus accumbens: involved with PLA2 activity and corticosterone secretion. Neuropsychopharmacology. 2007;32(3):736-744. doi: 10.1038/sj.npp.1301117 pmid: 16794572 
5. Lembke P, Capodice J, Hebert K, Swenson T. Influence of omega-3 (n3) index on performance and wellbeing in young adults after heavy eccentric exercise. J Sports Sci Med. 2014;13(1):151-156. pmid: 24570619

6. Hamazaki K, Itomura M, Huan M, Nishizawa H, Sawazaki S, Tanouchi M, et al. Effect of omega3 fatty acid-containing phospholipids on blood catecholamine concentrations in healthy volunteers: a randomized, placebo-controlled, double-blind trial. Nutrition. 2005;21(6):705-710. doi: 10.1016/j.nut.2004.07.020 pmid: 15925295

7. Ross BM. Omega-3 polyunsaturated fatty acids and anxiety disorders. Prostaglandins Leukot Essent Fatty Acids. 2009;81(5-6):309-312. doi: 10.1016/j.plefa.2009.10.004 pmid: 19906519

8. Serhan CN, Levy BD. Resolvins in inflammation: emergence of the pro-resolving superfamily of mediators. J Clin Invest. 2018;128(7):2657-2669. doi: 10.1172/JCI97943 pmid: 29757195

9. Black KE, Witard OC, Baker D, Healey P, Lewis V, Tavares F, et al. Adding omega-3 fatty acids to a protein-based supplement during pre-season training results in reduced muscle soreness and the better maintenance of explosive power in professional Rugby Union players. Eur J Sport Sci. 2018;18(10):13571367. doi: 10.1080/17461391.2018.1491626 pmid: 29985775

10. Panossian A, Wikman G. Pharmacology of Schisandra chinensis Bail.: an overview of Russian research and uses in medicine. $J$ Ethnopharmacol. 2008;118(2):183-212. doi: 10.1016/j.jep.2008.04.020 pmid: 18515024

11. Spedding S. Vitamin D and depression: a systematic review and meta-analysis comparing studies with and without biological flaws. Nutrients. 2014;6(4):1501-1518. doi: 10.3390/nu6041501 pmid: 24732019

12. Prapavessis H, Cox H, Brooks L. A test of Martens, Vealey and Burton's theory of competitive anxiety. Aust J Sci Med Sport. 1996;28(1):24-29. pmid: 8742863

13. Fernandes MG, Nunes SA, Raposo JV, Fernandes HM, Brustad R. The CSAI-2: An Examination of the Instrument's Factorial Validity and Reliability of the Intensity, Direction and Frequency Dimensions with Brazilian Athletes. J Appl Sport Psychol. 2013;25(4):377-391. doi: 10.1080/10413200.2012.744780

14. Vyncke K, Cruz Fernandez E, Fajo-Pascual M, Cuenca-Garcia M, De Keyzer W, Gonzalez-Gross $\mathrm{M}$, et al. Validation of the Diet Quality Index for Adolescents by comparison with biomarkers, nutrient and food intakes: the HELENA study. Br J Nutr. 2013;109(11):2067-2078. doi: 10.1017/S000711451200414X pmid: 23110799

15. Wilson PB, Madrigal LA. Associations Between Whole Blood and Dietary Omega-3 Polyunsaturated Fatty Acid Levels in Collegiate Athletes. Int J Sport Nutr Exerc Metab. 2016;26(6):497505. doi: 10.1123/ijsnem.2015-0316 pmid: 27098595

16. von Schacky C, Kemper M, Haslbauer R, Halle M. Low Omega-3 Index in 106 German elite winter endurance athletes: a pilot study. Int J Sport Nutr Exerc Metab. 2014;24(5):559-564. doi: 10.1123/ijsnem.2014-0041 pmid: 25203220

17. Tepsic J, Vucic V, Arsic A, Mazic S, Djelic M, Glibetic M. Unfavourable plasma and erythrocyte phospholipid fatty acid profile in elite amateur boxers. Eur J Sport Sci. 2013;13(4):414-421. doi: 10.1080/17461391.2011.630105 pmid: 23834548

18. Arsic A, Vucic V, Tepsic J, Mazic S, Djelic M, Glibetic M. Altered plasma and erythrocyte phospholipid fatty acid profile in elite female water polo and football players. Appl Physiol Nutr Metab. 2012;37(1):40-47. doi: 10.1139/h11-125 pmid: 22165902

19. Wilson PB, Madrigal LA. Associations among Omega-3 Fatty Acid Status, Anxiety, and Mental Toughness in Female Collegiate Athletes. $J$ Am Coll Nutr. 2017;36(8):602-607. doi: 10.1080/07315724.2017.1335249 pmid: 28910189

20. Panossian AG, Oganessian AS, Ambartsumian M, Gabrielian ES, Wagner H, Wikman G. Effects of heavy physical exercise and adaptogens on nitric oxide content in human saliva. Phytomedicine. 1999;6(1):17-26. doi: 10.1016/S0944-7113(99)80030-0 pmid: 10228607 